\title{
Developing a New Space over Deterministic Imaginary Time
}

\begin{abstract}
Mesut KAVAK*
In a day from days, when the famous $x$ is lengthened to $x_{2}$ and lost its virginity... Hey-o! Here comes the danger up in this club again. Listen up! Here's the story about a little guy, that lives in a dark world and uses power of wisdom as a torch to find way in darkness; and all day and all night and everything he sees is just illusion. I have been working about the laws of existence for a time [1-3]. I developed new formulas which were based on a strong mechanism over philosophical hypotheses. Nobody can answer easily; but I thought many times better mathematical infrastructure. Actually at the beginning, I noticed, that a fixed observer does observation of moving bodies being the bodies do a circular motion because of emerging and changing angles over time even if the objects move parallel manner relatively to the observer at that time. This would not happen accidentally even if abstract math says, nothing is going to change. Eureka! Finally while I was in a cafe today, I remembered and developed in a few hours a new method on some note papers which I demanded from cafe to explain existence, and thereupon I asked to my friends for leave, and I am writing towards morning in the name of giving a shoulder to the tired giants. The ancients smile on me!
\end{abstract}

\section{Primal Pledge}

There exist many exact proofs about the rules of right triangle in abstract math; but what are the actual geometry and mechanism in real physical medium? What an undertaking can give a triangle about existence to understand it? Actually, it is able to explain everything.

\section{X-Ray Images of the Secret Geometry}

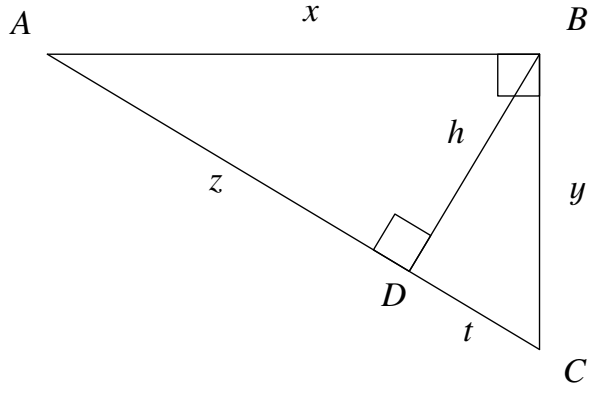

Fig. 1: Here is that famous right triangle about to be more famous. Actually any type triangle without any emerging right angle in it can be used instead; so also the others are going to be more famous soon.

There is a presentation of a right triangle on Fig. 1. Just think, that $x$ is lengthened to any $x_{2}$ value being the right angles are the same. Here, $B$ is a point which its coordinate in space is known. $C$ is the place an observer takes place in space. If $A$ is, it is a moving body. You have already understood and have seen the secret geometry; but even so, I am going to tell it more detailed.

Over the inequality and definition of $x_{2}>x$, it becomes (1) over the inequality of $h_{2}^{2}-h^{2}>z^{2}-z_{2}^{2}$,

$$
1>\frac{z^{2}-z_{2}^{2}}{h_{2}^{2}-h^{2}}
$$

where $h^{2}+z^{2}=x^{2}$ and $h_{2}^{2}+z_{2}^{2}=x_{2}^{2}$ are the equations over Pythagorean theorem. In the same manner, it becomes (2),

$$
h^{2}+t^{2}=h_{2}^{2}+t_{2}^{2}
$$

where $h^{2}+t^{2}=y^{2}$ and $h_{2}^{2}+t_{2}^{2}=y^{2}$ are the equations over Pythagorean theorem. If (2) is edited, it becomes $t^{2}-t_{2}^{2}=$ $h_{2}^{2}-h^{2}$; thus if $t^{2}-t_{2}^{2}$ is used instead of $h_{2}^{2}-h^{2}$ on (1), also it becomes (3) over $t^{2}-t_{2}^{2}>z^{2}-z_{2}^{2}$ inequality.

$$
1>\frac{z^{2}-z_{2}^{2}}{t^{2}-t_{2}^{2}}
$$

Now the actual displacement inequalities have been determined. Right this point, assume, that there is no displacement namely no lengthening. For this condition, it becomes $x_{2}=0$, $t_{2}=0$ and $z_{2}=0$; thus (3) becomes (4),

$$
t^{2}>z^{2}
$$

and (1) becomes (5).

$$
-h^{2}>z^{2}
$$

Hence it can never be $z=t$; thus it means

- Even if area would exist, exactly there is no middle point place; so a motion is always circular for any force applied.

- A right angle cannot emerge. It is only close to right angle due to the energy which area holds.

- It cannot be drawn two line segments which have the same length from a point in space to other two points. Namely, 3 or more objects cannot take place in space being the distance between each of them is the same. There is a time difference between each point of space at the same assumed global time. It also means, that each point has different speed for the same time interval. That skew of pyramids may not be mastery mistake. Instead, maybe is required by mastery.

- For $z \neq t$, it also becomes $x \neq y, h \neq z, h \neq y$ and $h \neq x$. Namely, while $x$ is lengthened, $y$ cannot protect its actual length. The medium is conservative. This also means, that length and thus 1 dimension do not exist alone; because 2 objects cannot take place at a distance relatively to each other.

- The shortest distance between 2 objects is not a line segment. This distance is an arc very close to a line segment.

- Parallel two line segment cannot be drawn beyond drawing line segment. They are exactly intersected, and the intersection point occurs according to area multitude of conservative area. By this way, no object can protect its speed. There is always an opposite space force to decelerate. By this way, as the same is acceptable for the existent smallest infinite part as well, constant speed motion or constant acceleration motion 
is not possible. Motion emerges by parts, and is always with small acceleration parts has frequency changing over time, and the result is average as assumed constant speed, and is seen as constant speed.

- A closed curve is not possible. Only infinite space closes curve.

- Motion cannot be independent of medium whatever the distance between moving bodies. This means, that there is an absolute entanglement between area and lengths in area although there is time differences. It shows one-piece behavior. This is only possible with emergence one by one. Namely, all mass is a single mass with emergence priority. Each point occurs by order. In this way, information is conserved forever; because flexible collision is not possible. There never occurs a mutual extinction which is because of decrease in the energy and mass by certain, whole numbers. Instead of it, during collisions, energy and mass values get smaller to infinite small part as irrational numbers; but it never disappears.

- There is always an emerging centrifugal force for motion because of circular displacement. In 3D, this may cause an outer space motion because of

$$
F=\frac{m v^{2} \sin (\alpha)}{r}
$$

and also is a good thrust engine potential can work in a closed environment especially is good for space, and by some transmission, it can store high energy by low energy, and you can produce thrust due to tensile strength of the used material, and can produce torque if it is connected around a second rotating wheel without angle. This motion can emerge because of an energy reduction which causes imbalance in motion which is the source of current total energy of objects.

- No object can be static. They always experience a potential difference since there is time difference and thus different densities over time, and move in the direction of lower density, more ordered area from disordered area. Also they always move in the directions of the one by one emergence since to be one of them before or after of the same two $F t$ work for the same force and time values as $+F t$ and $-F t$ changes displacement even for conservative energy and momentum.

- Over the law of induction, because of the time differences since any value of matter is different in each time interval during emergence constantly, the equation of

$$
\varepsilon=N_{2}-N_{1} \frac{\Phi_{2}-\Phi 1}{t_{2}-t_{1}}
$$

shows, that it is always $\varepsilon>0$ where $\varepsilon \in \mathbb{R}$. It means, that a coil even placed at infinite distance from a magnetic field has a voltage difference between the two top of the wire. If there is a fixed matter in the core, voltage will increase. If there is a fixed magnet in the core, then even if also the coil is fixed, the voltage is going to greatly increase; so it means, that even by switching two fixed coils have permanent magnet core and have different resistances will cause magnetic flux change; because electrons' orbital will slide even a current will not emerge, that it can emerge by required assembly and switching repeat. Maybe we can create a micro generator like producing a CPU that sheets between two permanent magnets will be connected serially to create high voltage difference to create electrical current beyond sliding orbitals. Free energy and thus self-running devices are possible since total energy of the medium is conserved. This is a focus, and you make matter worked by its own total energy. You must use more material. Namely, the same amount material generate more electricity by classical methods which require an external energy resource.

\section{Progressive Image}

For lengthened hypotenuse, over the inequality of $\left(z_{2}+\right.$ $\left.t_{2}\right)^{2}>(z+t)^{2}$ where $x_{2}+y^{2}=\left(z_{2}+t_{2}\right)^{2}$ and $x^{2}+y^{2}=(z+t)^{2}$, it becomes (6) for $x_{2}=0$.

$$
0>(z+t)^{2}
$$

It means, that $z+t \notin \mathbb{R}$. If you assume, that $z+t=0$, it becomes $0>0$; thus actually non of them can be 0 . It means, that even if there are no lengthening and thus motion, there were already area and motion. They are deterministic and cannot be $0 . z+t$ is always an imaginary number for the condition of (6).

In accordance with conservative space, over the components of the right triangle, it becomes $x+y+m=n$ and $x^{2}+y^{2}=m^{2}$ where $m=z+t$; thus becomes $\tau+t+m=n$ and $\tau^{2}+t^{2}=m^{2}$ where $\tau$ and $t$ are time here, and is $m=\sqrt{\tau^{2}+t^{2}}$. It seems, that $x$ and $y$ or $\tau$ and $t$ cannot take random values, that they take certain values according to a rule. If $\tau+t+m=n$ is edited, it becomes $(\tau+t)^{2}=(n-m)^{2}$ and thus it becomes $\tau^{2}+t^{2}+2 \tau t=n^{2}-2 n m+m^{2}$. Since is $\tau^{2}+t^{2}=m^{2}$, finally it becomes (7),

$$
2(\tau t-n m)-n^{2}=0
$$

where $n=\tau+t+\sqrt{\tau^{2}+t^{2}}$. For this equation of (7), the roots become (8) as imaginary time since it cannot be 0 over (6).

$$
\tau=\mp i t
$$

As constant speed and so $x=v t$ is not possible, because of $x=a t^{2}$, when real time is emerged, the distance and time emerge in opposite ways over the time on $x=a t^{2}$, and know, that image appears as illusion in 3D because of the complex roots and thus complex plane which is perpendicular to $(x, y)$ plane. After that, if you want to express the motion as average, you do not have to use imaginary time since is real time after this, just use its multitude on $x=v t$ and it becomes $x=-v t$.

Assume, that $x$ is lengthened by $k$, and then also $t$ is lengthened automatically because of changing geometry on Fig. 1. For this condition, as is $x \neq t$, also it becomes (9),

$$
\frac{n_{2}-n_{1}}{x} \neq \frac{n_{2}-n_{1}}{t}
$$

for two consecutive increase in $k$, where $\frac{x+k}{x}, \frac{t+k}{t}, x_{2}=x+k$ and $t_{2}=t+k$. Namely, suck an increase is not possible. If $x$ changes by a number like $k$, then $t$ is going to change by different number. It does not happen in the same ratio. Namely, it accelerates in a conservative area. As the exact opposite is also possible, constant speed motion is not possible. Namely, while you are assuming $x$ is lengthened by constant speed, since $t$ is going to accelerate, for an assumed small time interval, also you can assume that I did not want to move $x$, I just wanted to move $t$, and it accelerated. By this acceleration amount, you will see that also $x$ is accelerated. Namely, these happen at the same time, by acceleration. One element of area cannot be independent of another. namely, if you say $x$ moved at constant speed, this is only assumption and is an average speed to calculate.

According to these information, it can be said

- There is a single work done to create all the area because of the entanglement and single mass; so each part of space has the same speed as average. None of them 
can go faster than another one. No point could gain infinite energy because of the time differences, since the work done is not infinite, is done over time, is done at a frequency, as the work done is equal to kinetic energy.

- There is always a space tension since points experience potential difference which is because of emerging different densities over time. Points want to move towards lower density; so also matter is incompressible. It cannot be compressed down to infinite small part. Speed of the work done and frequency of emerging density difference determines this base compressing limit. More energy is required to create denser and thus more disordered area. We can explain it such, that in accordance with conservative area, over the triangle, for a function of $f(x)=a x$, it becomes (10) over (10a) where $x_{2}=x \mp k$

$$
\begin{gathered}
x=\frac{\mp k}{\sqrt{2}-1} \\
\int a x d x=\int_{x}^{x_{2}} a x d x
\end{gathered}
$$

Namely, the increase does not happen randomly. You need more speed of the work done to create the area and so need taller $x$. This creates a space tension always for both compressing and lengthening. There is going to be always a resistance and a limit for the both.

Here, important thing is to change the geometry of the triangle into another shape being the area is the same; so even if this calculation is going to create a trapezoid and a triangle in a wider triangle, both of them are going to have the same area being $x$ is a constant. There is no difference.

Warning

- Over (10), it can be said, that $x$ is always irrational This is pretty suitable since there are always potential and time differences even for any short interval of any short interval. If also matter could not gain infinite energy and area because of the finite work done, it means, finite matter is virtual part of infinite or infinite information. All infinite information is certain only. This irrationality cannot happen at the same time. Namely an irrational number cannot be inclusive of works with its all infinite digits after the comma; but its limit may constantly change. This creates time and thus motion again and again, and also you cannot say is constant speed since is uncertain.

\section{The Imaginary Real-like Strange Such and Such Re- sult}

In accordance with these information, as a result, matter is deterministic. Also it is uncertain as its any measurable value is also uncertain. It has no infinite energy and thus volume. It emerges at a frequency. Right this point, to calculate, there emerge some local and global min. and max. points and threshold values as a reference. Namely, if you make a calculation for centrifugal force over circular displacement, you cannot say 'Alas! The force became infinite, hide behind a stone!' since $\tan (\alpha)$ became 90 according to a reference. It does not happen such. Angle is always fixed even if is irrational; but the same assumed small motion as a reference and threshold value repeats. There is no infinite mass or standing time in black holes or somewhere else in the universe.

For example, since kinetic energy is equal to work done, a stopper opposite limited work on the infinite brings matter total energy out at the amount of this opposite enforcement. Since matter always experiences potential differences, it always moves to lower density and thus more ordered place, and then it again experiences a denser space. This always repeats itself as long as there is a motion and area. Since to be one of them before or after of the same two opposite $F t$ works in space as $+F t$ and $-F t$ changes the displacement even momentum and energy will be conserved at that time, if matter is formed by a circular motion, there must be always a potential difference or initial movement for the resultants on Fig. 2 to emerge of motion if you assume the motion closes a perfect circle, that actually does not close, that is not important since the basic logic is going to be the same.

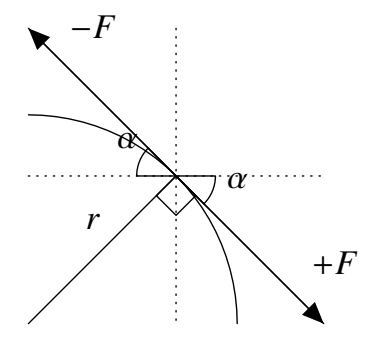

Fig. 2: The resultants

Any way, according to the above stated information, we must leave one of the resultants like on Fig. 2 to emerge of matter to calculate it. It will be enough for the calculation. Being $m v r / r=\tan (\alpha)$, for $m v r / r=\int \tan (\alpha)$ equation it becomes Eq. (11) for 1 second.

$$
\log _{e} \cos \alpha=-m v
$$

As required by uncertainty and one by one emergence, mass decreases at the same ratio with time [1] for the smaller slices of time directly proportional; because wave packet or in the other name assumed existent smallest part of matter repeats itself; but the smallest part may emerge in different ratios with time; so for $\cos ^{-1}\left(e^{-m v t}\right)=\alpha$ by decreased mass, $\frac{2 \pi r \alpha}{360}=v t$ and $h v=m v^{2}$ equations, Eq. (11) turns into Eq. (12) as the below,

$$
\log _{e} \cos \alpha=-\frac{\pi r \alpha m}{180}
$$

where $v$ is any multitude formation speed as light speed, and it can only be solved by solving equation as an irrational value, as actually this irrationality is required by uncertainty as well.

This angle on Eq. (12) is the angle emerges in the smallest time as required by uncertainty as a threshold value. For this angle, the smallest time in the other name the Planck Time can be found out over $\frac{2 \pi r \alpha}{360}=v t$ and the smallest distance can be found out over $\frac{2 \pi r \alpha}{360}$ as the Planck Length. As you can see, the angle changes according to the multitude of Planck constant but being zero or 90 .

Or for example, if you leave a handful of electrons to a nucleus, it only choose some of them, and the electrons only get certain orbitals or actually get some orbitals they exist more. They cannot exist there randomly. In the same manner, galaxy spiral arms do not take place accidentally. If you place some marbles which have the same mass and density in space being the distance between them is the same, you will see, that they do not come together at a point. Instead of it, according to emerging way which is because of the time differences, they are grouped; but maybe according to emergence way or in the other name spin of the universe, some of them are grouped 
slower. At the end, again each group create a second group. This continues until coming together. Namely as a result, like in the double slit experiment, whatever the frequency or type of the rays, if light passes through such a slit, the image appears at the screen being some areas are denser even if the light will touch the other points between the denser lines as well.

\section{Acknowledgment}

I shall not demand patent right. Anybody who wants to use the above stated things can use freely without asking.

At the beginning, once I could not sleep, while I was thinking about energy in my bed after midnight. I was smoking in darkness, and was spinning my cigarette. I thought, that is it possible to brighten the environment by spinning the cigarette faster for the same light energy. Namely, can external enforcement turn into light energy. I wrote an equation like $m_{0} c^{2}+m v^{2}=m c^{2}$, and after that any thing is opened for me. I published my papers after this $[1,2]$. Last week, after midnight just before sleeping, I noticed in my bed, that free space itself can cause a parachute effect on the moving bodies especially the bodies move in low gravitational fields like in Pioneer Anomaly due to the density of the object, and upon this, I published a paper about this [3]. Finally I could built a new, short, understandable and simple infrastructure at the expense of sleeplessness now as well. In the name of any theoretician, by the text which had become interpreter of our feelings, as the poet had said:

This is nightlife,

We dance under the moonlight,

And party till we feel high,

We're ready for the good time,

All over the nation,

We have a celebration,

And party till we feel high,

We're ready for the good life.

It is very nice, listening it makes me happy, even the reinterpretation of Korpiklaani which sounds like Chechen music is enjoyable as well, I would like to use Ievan Polkka; but I have no idea what they talk about.

Additionally, I do not know am I going to work on these; but Navier-Stokes Equation and Mass Gap of Yang-Mills may be solved soon by some detonators, and may write in front of the problems 'exploded' in claymath website.

Just one more thing, that actually, in topological perspective, there is no difference between a torus and a sphere since a closed curve is not possible. Actually, it means, that there is no difference between shapes. This means, relativistic topology must emerge somewhere or you cannot define shape.

Goodbye!

\section{References}

1. Kavak M. 2018, Complement Inferences on Theoretical Physics and Mathematics, OSF Preprints, Available online: https://osf.io/tw52w/

2. Kavak M. 2016, On the Uncertainty Principle, American Journal of Physics and Applications, Vol. 4, No. 4, 2016, pp. 90-123. Available online: https://osf.io/t8zqw/

3. Kavak M. 2018, Short Note on Space Wind Powered by Disorder: Dark Energy, OSF Preprints, Available online: https://osf.io/nqswk/ 